Modern Electronic Technology

https://ojs.s-p.sg/index.php/met

\title{
Hybrid Gait Planning of A Hexapod Robot
}

\section{Ruyi Wang* Neil Powell}

Department of Electronic and Electrical Engineering, The University of Sheffield, Sheffield, S10 2TN, UK

\begin{tabular}{l}
\hline ARTICLE INFO \\
\hline Article history \\
Received: 29 June 2020 \\
Revised: 6 July 2020 \\
Accepted: 9 October 2020 \\
Published Online: 16 October 2020 \\
\hline Keywords: \\
Hexapod \\
Tripod \\
Transverse gait \\
Swivel gait
\end{tabular}

\begin{abstract}
The adapotation of gaits pattern is a basic and important for the hexapod robot to move stably and efficiently, which depends on the servos of the robot's legs, and also the body structure of the robot.

This paper compares the tripod gait and the crab-inspired gait for a specific hexapod to move forward and move backward; turn left and turn right and integrates the two gaits to apply them under different conditions. The hexapod has three servos on each legs, thus the freedom level of each leg is three-degree. From the comparative experiment, this two gait patterns are suitable for different turning demands.
\end{abstract}

\section{Introduction}

$\mathrm{C}$ ompared with wheeled robot and tracked robot, legged robots have stronger ability to rough terrain and is more flexible in complex environment of disaster rescue and terrain exploration ${ }^{[2]}$. The hexapod robot has better stability and load capacity among the legged robot, which can achieve various applications in different scenarios, such as lunar exploration, nuclear power plant inspection, etc., with a wealth of gait modes ${ }^{[1,3]}$. Thus the hexapod is required to keep strong stability and high adaptability. In addition, considering power supply and efficiency, it also needs to coordinate limb and move effectively. Moreover, the torso of the hexapod robot can withstand certain losses, such as in the absence of a leg, it can also complete some commands. Therefore, excellent hexapod design should consider the mechanical structure, gait control, robot autonomy, multi-sensor integration and power supply of the robot.
According to the torso of the hexapod and the distribution of legs, there are rectangular hexapod and hexagonal hexapod, of which each leg is better to have three degrees of freedom ${ }^{[7]}$. Based on the structure, the movement of the hexapod needs to analyze the trajectory of each leg and inverse its kinematics to calculate steering angles of each motion on legs at real-time ${ }^{[9,11]}$.

However, due to the limitations of the computing power and efficiency of the movement, it is necessary to optimize gait algorithms. Although there are several of advanced algorithms about gait, such as neural network, genetic algorithm and artificial intelligence algorithm, the most common and basic gait control is the tripod. The tripod gait of the hexapod refers to the movement of three legs at the same time, which can achieve fast movement with stability ${ }^{[4]}$. Many studies have verifies the superiority of the tripod. However, the general tripod lacks adaptability in steering ${ }^{[8]}$, proposed that each leg is regarded as an independent system of the kinematics model of the

*Corresponding Author:

Ruyi Wang,

Department of Electronic and Electrical Engineering, The University of Sheffield, Sheffield, S10 2TN, UK;

E-mail: rwang46@sheffield.ac.uk. 
gait, and illustrated the crab-inspired lateral movement to move left and move right ${ }^{[5]}$. The two gaits are suitable for different scenarios.

To optimize gait of the hexapod, this paper initially describes the mechanical structure of the robot, then analyze its kinematics on this basis. In this paper, a modular algorithm is proposed to reduce the computation consumption for move forward and backward. In addition, this paper optimized a turning gait of the hexapod.

\section{Structure \& Kinematics}

\subsection{Hexapod Structure}

\subsubsection{Mechanical Configuration}

The experimental in this article is a rectangular hexapod robot BH-3 of Lynxmotion in Figure 1, of which each leg has three degrees of freedom.

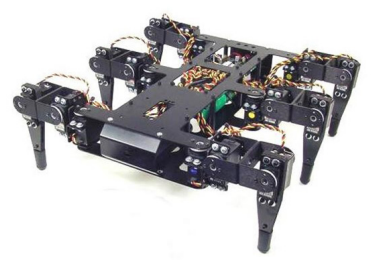

Figure 1. BH-3 Hexapod

Figure 2 shows the structure model of the hexapod and the dynamic model of a single leg. In the models, the appropriate coordinate systems are introduced, and the size of each part is marked to establish and appropriate inverse kinematics model. The movement range of each joint is recorded in table 1 .

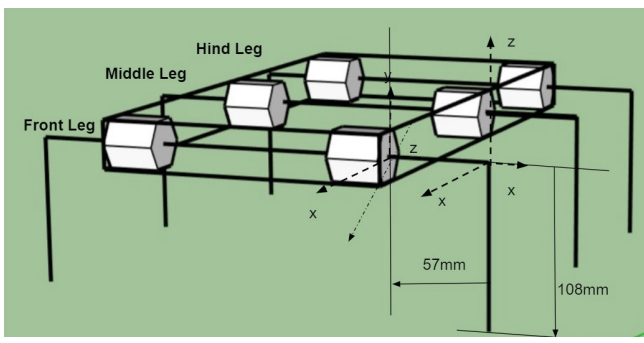

(a)

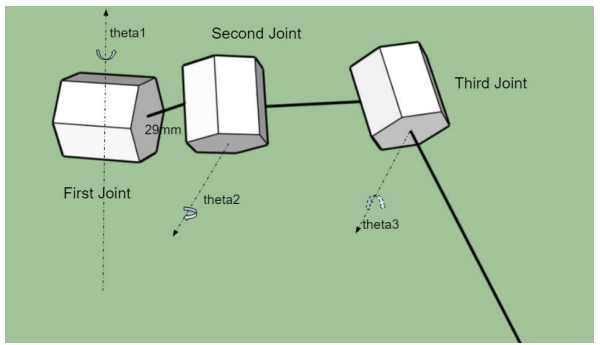

(b)

Figure 2. Hexapod Configuration
Table 1. Move Range of Each Joint

\begin{tabular}{|c|c|c|c|}
\hline & Front & Middle & Hind \\
\hline $1^{\text {st }}$ Joint & {$\left[-\frac{\pi}{2}, \frac{\pi}{2}\right]$} & {$\left[-\frac{\pi}{3}, \frac{\pi}{3}\right]$} & {$\left[-\frac{\pi}{2}, \frac{\pi}{2}\right]$} \\
\hline $2^{\text {nd }}$ Joint & {$\left[-\frac{\pi}{4}, \frac{\pi}{2}\right]$} \\
\hline $3^{\text {rd }}$ Joint & {$\left[-\frac{3 \pi}{4}, 0\right]$} \\
\hline
\end{tabular}

\subsubsection{Electronic Hardware}

This hexapod is equipped with the SSC-32U servo controller, which supports preloading code and reading the current position and speed of servos, and 18 HS-645MG standard servos. The hexapod changes the rotation angle of each motor to control the joint through code commands to mobilize legs and to move body. In addition, Bluetooth module is added to the hexapod that it can be controlled by communicating with computer.

\subsection{Inverse Kinematics}

For the motion control of the hexapod, the inverse kinematics of its leg is mainly studied. In a tripod gait, the hexapod maintains three supporting legs at any time, keeping its body parallel to the ground. Due to the kinematics principle and trajectory of each leg of the hexapod are the same or the angle is opposite, we can analyze and calculate the motion of one leg, so as to establish the leg cooperation to mobilize the movement of all legs, so as to simplify the overall kinematics complexity.

\subsubsection{Coordinate System}

According to the coordinate system and angle shown in Figure 3, taking the right front leg as an example, the angle values of three servos (theta1, theta2 and theta3) on the leg can be calculated.

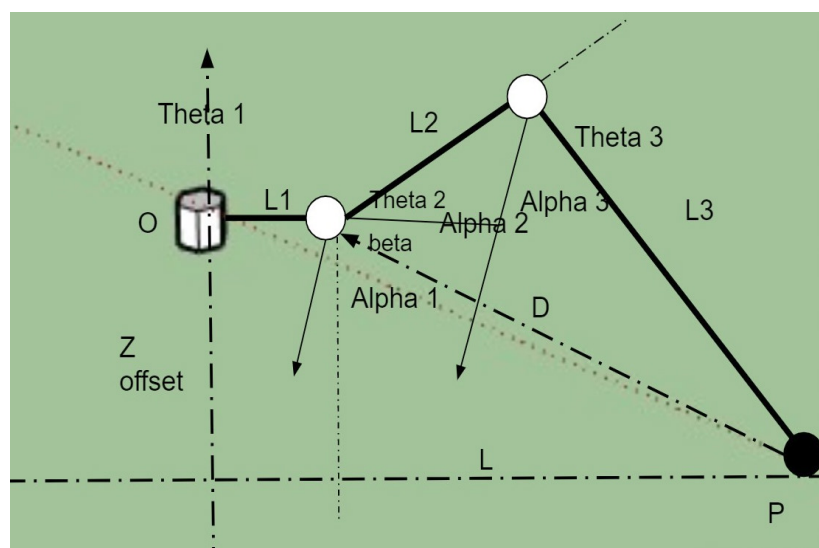

Figure 3. Single Leg Coordinate 


\subsubsection{Inverse Kinematics for Tripod Gait}

$\theta_{i}=\tan ^{-1} \frac{y_{i}}{x_{i}}, i$ is the number of servo on each leg.

Unlike $\theta_{1}$, which can be calculated directly, $\theta_{2}$ and $\theta_{3}$ have to calculate angles $a_{1}, a_{2}$ and $a_{3}$ preliminarily, which are described in Figure 3.

$$
\begin{gathered}
\alpha_{1}=\tan ^{-1}\left(D / Z_{0}\right), \\
\alpha_{2}=\cos ^{-1}\left(\frac{L_{3}^{2}-D^{2}-L_{2}^{2}}{-2 L_{2} D}\right), \\
\alpha_{3}=\cos ^{-1}\left(\frac{D^{2}-L_{3}^{2}-L_{2}^{2}}{-2 L_{2} L_{3}}\right)
\end{gathered}
$$

\section{Gait Design}

\subsection{Tripod Gait}

The tripod gait of the hexapod is presented in Figure 3. The hexapod transfers three legs to lift or to slump. Thus the six legs are divided into two groups, which switch alternately. During the movement, there are always three legs on the ground as support legs.
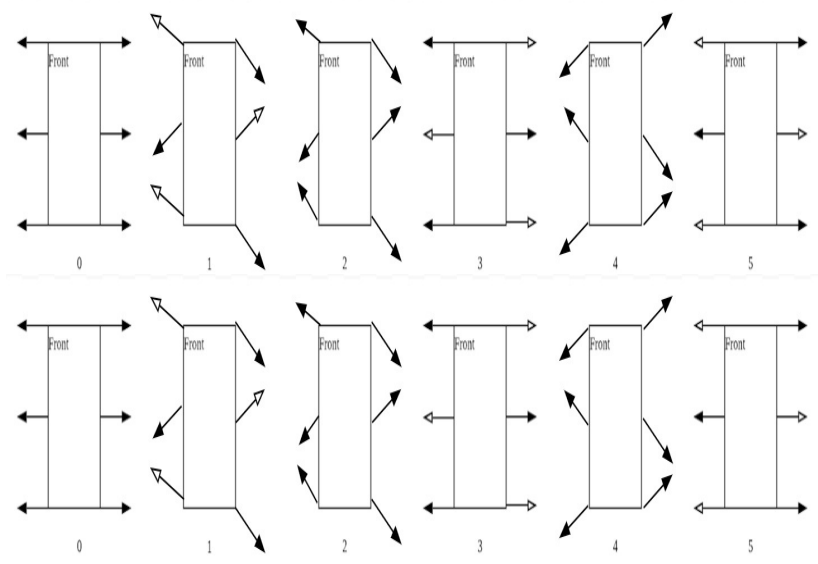

Figure 3. Tripod Gait of Forward Movement

To ensure the stability and reliability of the movement, arbitrary two adjacent legs of the hexapod are not allowed to leave the ground at the same time ${ }^{[6]}$, thus all supporting legs form a stable triangle structure ${ }^{[12]}$. In Fig 3 the hollow arrow represents swing legs and the solid arrow represents supporting legs. The hexapod can move forward $22 \mathrm{~cm}$ stably each swing.

The sequence of moving legs forward and backward for tripod gait is the same, the original hind leg can be regarded as the front leg that the backward movement can be completed as Figure 3.

Table 2 illustrates the position of legs for moving for- ward and backward.

Table 2. Position State

\begin{tabular}{|c|c|c|}
\hline State $(\mathrm{x}, \mathrm{y}, \mathrm{z})$ & Forward(mm) & Backward(mm) \\
\hline Lift & $(0,0,15)$ & $(0,0,15)$ \\
\hline Swing & $(0,110,15)$ & $(0,-110,15)$ \\
\hline Support & $(0,110,0)$ & $(0,-110,0)$ \\
\hline
\end{tabular}

\subsection{Turning Gait Planning}

The gait of the hexapod changes greatly when turning, where the mechanical structure, steering frequency and the path of the hexapod should be considered comprehensively. The first joints of BH-3 hexapod can rotate at right angles, thus a specific swivel gait is proposed to realize high-speed turning. Moreover, this 3.2.1 Swivel Gait

According to Figure 4, the hexapod moves legs 6 times for once turning, which is less twice than conventional tripod gait. During the turning, two adjacent legs are moved together. However, there are always three supporting legs. In addition, the angular symmetry of the legs provides steering force while maintaining stability, which can be applied to this rectangular hexapod.

Each movement in Figure 4 needs 1 seconds based on initial setting, thus it costs 6 seconds to complete swivel.

The principle of turning left and turning right is exactly the same, but the opposite leg should be mobilized to provide steering force and support the stability of the torso.

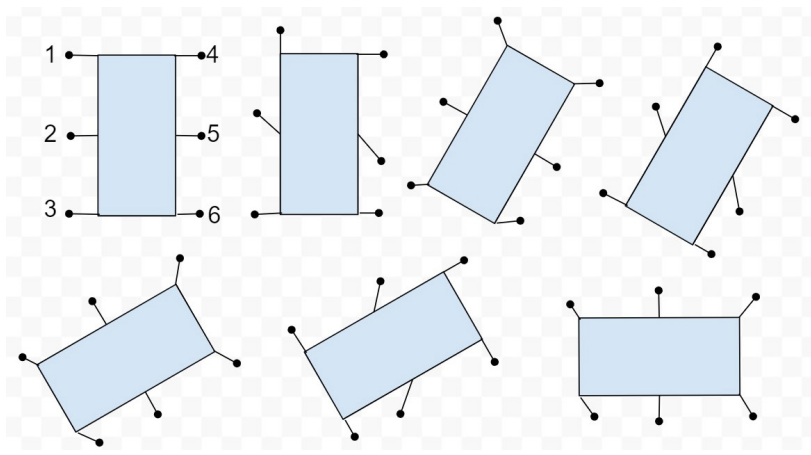

Figure 4. Swivel Gait of Turning Right

The changed position is presented in Table 3 with the rotation angle of joints.

Table 3

\begin{tabular}{|c|c|c|}
\hline Joint State $\left(1^{\text {st }}, 2^{\text {nd }}, 3^{\text {rd }}\right)$ & $\left.\operatorname{Turn~Left(~}^{(}\right)$ & Turn Right $\left({ }^{\circ}\right)$ \\
\hline lift & \multicolumn{2}{|c|}{$(0,15,-45)$} \\
\hline Leg 1 & $(90,15,-45)$ & $(30,15,-45)$ \\
\hline Leg 2 & $(30,15,-45)$ & $(-30,15,-45)$ \\
\hline Leg 5 & $(-30,15,-45)$ & $(30,15,-45)$ \\
\hline Leg 3 & $(30,15,-45)$ & $(-30,15,-45)$ \\
\hline Leg 6 & $(-30,15,-45)$ & $(30,15,-45)$ \\
\hline Leg 4 & $(30,15,-45)$ & $(90,15,-45)$ \\
\hline
\end{tabular}




\subsubsection{Transverse Gait}

Inspired by the walking pattern of crabs, the robot can walk horizontally ${ }^{[7]}$, both move to right and left without turning, which is more conducive to short-distance and rapid movement of the rectangular hexapod, due to the rectangle is not fully symmetric that it is inconvenient for swivel gait. Thus using transverse gait can improve the efficiency and reduce energy cost to move to left or right for some situations, which is presented in Figure 5.
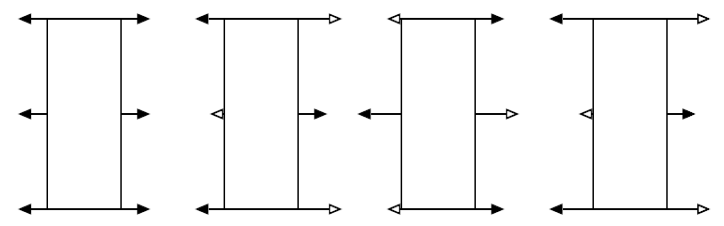

Figure 5. Transverse Gait of Hexapod

The transverse gait mainly relies on movement of the third joints on each leg. The movement of leg2 requires the third Joint on it which changes $-15^{\circ}$ once. In contrast the leg3 should be change when the hexapod move left.

Table 4

\begin{tabular}{|c|c|c|}
\hline State $(\mathrm{x}, \mathrm{y}, \mathrm{z})$ & $\operatorname{Right}(\mathrm{mm})$ & $\operatorname{Left}(\mathrm{mm})$ \\
\hline $\operatorname{leg} 1, \operatorname{leg} 3, \operatorname{leg} 5$ & $(0,0,0)$ & $(-30,0,15)$ \\
\hline Leg2, leg4, leg6 & $(30,0,15)$ & $(0,0,0)$ \\
\hline
\end{tabular}

In addition, since the hexapod has no gyroscope, it should executes the offset after each phase of motion.

\section{Experiment \& Result}

\subsection{System Design of Hexapod Robot}

Gait control code is preloaded onto the servo controller. In addition, a controller is generated with MATLAB, which can control the hexapod to move, stop and offset. The hexapod communicates with computer via Bluetooth module, of which the computer sends commands to the servo controller and the hexapod executes responding instructions.

For the experimental hexapod, the SSC-32U transfer the augment into transitional time of the position. For example, it takes 10 seconds to move $90^{\circ}$ if the speed value is 100 us per second. Considering the efficiency, the pulse width is defined as 1800 microseconds and the speed value is 1000 microseconds.

\subsubsection{Straight Gait}

In the test of the forward and hind legs of the hexapod robot, two gait control methods are compared in the same road section. In the first method, the six legs of the hexapod robot are divided into two groups by using the joint control method. The two groups of legs turn into supporting legs and swinging legs. The inverse kinematics of one leg in each group is calculated, and the other legs directly or reversely quote according to the angle obtained from the calculation results, so as to realize cooperative control. The second method is to calculate the inverse kinematics of six legs, and call each leg separately. According to results of the experiment in table 5 , it is obviously that the first method is much more efficient for the hexapod to walk on flat terrain. In addition, the speed and dexterity of the hexapod forward and backward are almost the same.

Table 5. Test Results for Method 1 \& Method 2 to Move for $50 \mathrm{~m} \& 100 \mathrm{~m}$

\begin{tabular}{|c|c|c|}
\hline Flat Path & $50 \mathrm{~m}$ & $100 \mathrm{~m}$ \\
\hline M1 Forward & $8^{\prime} 22$ & $13^{\prime} 59$ \\
\hline M2 Forward & $9^{\prime} 54$ & $19^{\prime} 28$ \\
\hline M1 Backward & $8^{\prime} 49$ & $14^{\prime} 09$ \\
\hline M2 Backward & $9^{\prime} 58$ & $19^{\prime} 26$ \\
\hline
\end{tabular}

\subsubsection{Turning Gait}

The hexapod is tested for different path planning in the two paths shown in Figure 7. Different gait designs for 
path a) and path b) are recorded in Table 6 and Table 7 .

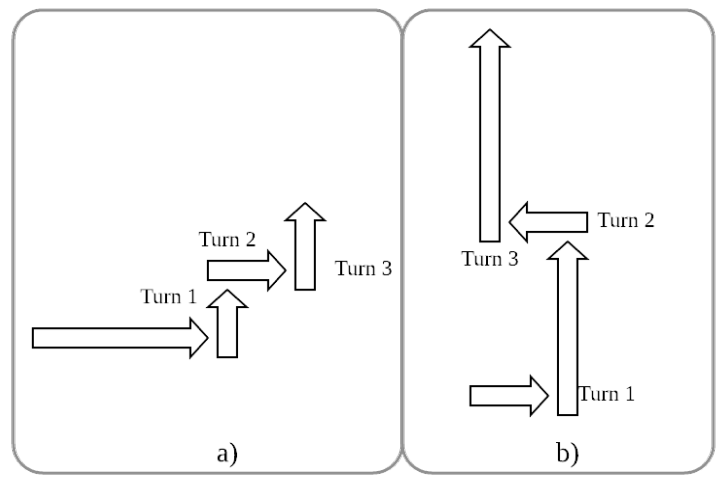

Figure 7. Test Path of Gaits

Table 6. Timing Results for Path a)

\begin{tabular}{|c|c|c|c|}
\hline a) & 1 & 2 & 3 \\
\hline Turn 1 & Swivel & Transverse & Transverse \\
\hline Turn 2 & Swivel & Forward & Transverse \\
\hline Turn 3 & Swivel & Transverse & Swivel \\
\hline Time & $8 ’ 29$ & $5{ }^{\prime} 37$ & $5{ }^{\prime} 20$ \\
\hline
\end{tabular}

Table 7. Timing Results for Path b)

\begin{tabular}{|c|c|c|c|}
\hline b) & 1 & 2 & 3 \\
\hline Turn 1 & Swivel & Transverse & Swivel \\
\hline Turn 2 & Swivel & Backward & Transverse \\
\hline Turn 3 & Swivel & Transverse & Swivel \\
\hline Time & $11^{\prime} 02$ & $13^{\prime} 31$ & $10^{\prime} 12$ \\
\hline
\end{tabular}

Obviously, the short-distance steering is more suitable for using the transverse gait, while the long-distance steering is more suitable for the swivel gait.

\section{Discussion}

\subsection{Tripod Gait}

The motor rotation angle of the other two legs can be calculated by the trajectory of any leg in the front leg, which reduces computing complexity. However, such a calculation method is not flexible in highly complex terrain.

\subsection{Turning Gait}

The steering gait proposed in this paper can efficiently complete 45 degree turning and 90 degree turning, and it costs less computing and energy consumption than high frequency steering of 5-degree by 15 -degree.

The transverse gait is unsuitable for non-90 degree steering, due to it is difficult to maintain stability in the situation. However, the transverse gait is slower than straight tripod gait, and increases computing complexity. Thus the hexapod should adopt the swivel gait when there is long-distance movement after turning as Figure 7(a), the transverse gait can be implemented for frequently turning in Figure 7 (b).

Thus the two gait can be combined together for turning.

\section{Conclusion}

This paper introduces the configuration and kinematics of the hexapod, and proposes a special tripod gait control, which determines the trajectories of the other two legs according to the gait planning of one leg. The design reduces computing consumption. Furthermore, a simple swivel gait is proposed and combined with the transverse gait. Thus the hexapod is manipulated to turning and to move smoothly. The experiment result indicates that the hexapod can move following command with designed gait. The gait is suitable for most hexapod robots, and requires less power consumption than conventional method.

\section{Future Work}

The gait patterns in this paper is convenient for moving forward, backward and turning on known roads, but the motor rotation angle obtained by the inverse kinematics method lacks flexibility and efficiency. In addition, the tripod is short of adaptability to unknown complex terrain and obstacle, where the reactive gait planning can improve it efficiently. In the future, reactive gait planning can be achieved by introducing genetic algorithms or central pattern generator into gait design.

\section{References}

[1] Carbone, Giuseppe, and Di Nuovo, Alessandro. "A Hybrid Multi-objective Evolutionary Approach for Optimal Path Planning of a Hexapod Robot a Preliminary Study." 9668 (2016): 131-44. Web.

[2] Bai, Long, Hu, et al. "CPG-Based Gait Generation of the Curved-Leg Hexapod Robot with Smooth Gait Transition." Sensors (Basel, Switzerland) 19.17 (2019): 3705.

[3] Deng Hua et al. "Gait and trajectory rolling planning and control of hexapod robots for disaster rescue applications" Robotics and Autonomous Systems vol. 95 pp. 13-24 2017.

[4] G. Zhong, H. Deng, G. Xin and H. Wang, "Dynamic Hybrid Control of a Hexapod Walking Robot: Experimental Verification," in IEEE Transactions on Industrial Electronics, vol. 63, no. 8, pp. 5001-5011, Aug. 2016, doi: 10.1109/TIE.2016.2551679.

[5] Hasnaa, El Hansali, and Bennani Mohammed. "Plan- 
ning Tripod Gait of a Hexapod Robot.” 2017 14th International Multi-Conference on Systems, Signals \& Devices (SSD) 2017 (2017): 163-68.

[6] K. Ozyalcin, I. H. Akay, Y. Ozturk, et al, "New Design and Development of Reconfigurable-Hybrid Hexapod Robot," IECON 2018 - 44th Annual Conference of the IEEE Industrial Electronics Society, Washington, DC, 2018, pp. 2583-2588, doi: 10.1109/ IECON.2018.8591312.

[7] Liu, Yiqun, Fan, Xuanxia, Ding, et al, Haibo. "Fault-Tolerant Tripod Gait Planning and Verification of a Hexapod Robot." Applied Sciences 10.8 (2020): 2959.

[8] L. Xu, W. Liu, Z. Wang and W. Xu, "Gait planning method of a hexapod robot based on the central pattern generators: Simulation and experiment," 2013 IEEE International Conference on Robotics and Biomimetics (ROBIO), Shenzhen, 2013, pp. 698-703, doi: 10.1109/ROBIO.2013.6739542.
[9] Priandana, K., Buono, and Wulandari. "Hexapod Leg Coordination Using Simple Geometrical Tripod-gait and Inverse Kinematics Approach.” 2017 International Conference on Advanced Computer Science and Information Systems (ICACSIS) 2018 (2017): 35-40.

[10] Sun, Jianbo, Jie Ren, Yinglian Jin, et al. "Hexapod Robot Kinematics Modeling and Tripod Gait Design Based on the Foot End Trajectory." 2017 IEEE International Conference on Robotics and Biomimetics (ROBIO) 2018 (2017): 2611-616.

[11] Xingji Duan, Weihai Chen, Shouqian Yu, et al. "Tripod Gaits Planning and Kinematics Analysis of a Hexapod Robot." 2009 IEEE International Conference on Control and Automation (2009): 1850-855.

[12] Z. Kong, M. Xu, X. Wang, et al. Gait planning and gait transition of AmphiHex-I," 2014 IEEE/ASME International Conference on Advanced Intelligent Mechatronics, Besacon, 2014, pp. 66-71, doi: 10.1109/AIM.2014.6878048. 